\title{
Spin-orbit interaction in a generic vortex field transmitted through an elliptic fiber
}

\author{
C.N. Alexeyev", M.S. Soskin ${ }^{*}$, A.V. Volyar* \\ *National Tavrida V.I. Vernadsky University, \\ Yaltinskaya 4, Simferopol 95007, Crimea, Ukraine, tel.: (0652) 23-02-48, fax: (0652) 23-23-10, \\ e-mail:volyar@ccssu.crimea.ua \\ +Institute of Physics, National Academy of Sciences of Ukraine, Kyiv 252650, Ukraine, \\ tel.:(044)265-55-63,e-mail:msoskin@iop.kiev.ua
}

\begin{abstract}
Absract. Any small external perturbation on an ideal round optical fiber induces cross section deformation and transforms eigen guided vortices into generic vortex fields, which can change their structure and angular momentum. It is the properties of these vortical fields in an elliptic weakly guiding optical fiber that we consider in this paper. The eigenfunctions and the spectrum of polarization corrections to the scalar propagation constant in the case of relatively large and small values of a fiber ellipticity are obtained by means of the spin-orbit interaction operator method. Discussed is the conversion process of a spin and orbit angular momenta on a vortex propagation along a deformed fiber.
\end{abstract}

Keywords: optical vortex, elliptic optical fiber, spin-orbit interaction.

Paper received 09.06.00; revised manuscript received 08.09.00; accepted for publication 12.12.00.

\section{Introduction}

Optical vortices represent the unique form of electromagnetic field existence. The distinguishing feature of this form is the presence of spatial lines where both real and imaginary parts of wave function simultaneously equal to zero. The concept of an optical vortex as a scalar wave field with a pure screw wave front dislocation was first introduced by J. Nye and M. Berry in [1]. Since such a field in the vicinity of the dislocation has a phase singularity, the smoothness of its wave function requires the vanishing of its modulus. Helicoidal structure of the wave front is also an important attribute of the optical vortex $[2,3]$. For beams with such helicoidal wave front structure the phase circulation around the beam axis does not equal to zero. Strictly speaking, it was the property that have determined the term «an optical vortex». The number of helicoid's branches determines its topological charge 1 , while the direction of its rotation - the sign of the charge. More intricate vortical structures - paraxial spiral laser beams - have been studied in [4]. To generate optical vortices suitable for practical application there have been developed several original methods. In particular, the most widespread among them are the method of intro-cavity generation of paraxial beams [5] and the method of computer-generated holograms $[2,6]$. As it was demonstrated by further researches, a nonzero value of the phase circulation is closely connected with the orbital angular momentum $M_{z, 1}$ along the optical axis, while circular polarization of the beam induces its spin $M_{z, s}$ (where $s= \pm 1$ characterises the direction of electric field vector rotation) [2,7-10]. In the paraxial approximation the total angular momentum per a circularly polarized photon is equal to $\hbar(l+\sigma)$ [7].

In the paraxial approximation the polarization of the beam does not influence its propagation. Nevertheless, the properties of the paraxial optical vortex depend both on its topological charge $l$ and polarization $\sigma$. In general, the polarization of electromagnetic wave is a function of coordinates possessing points, lines and surfaces of singularity. The properties of polarization singularities disclinations - have been studied in details in [11-13].

The presence of optical vortices in the output radiation of a multimode fiber was first revealed by B.Ya. Zel'dovich and N.B. Baranova in 1980 [4]. In the fundamental work of A. Snyder and J. Love [15], there was mentioned the possibility of an alternative representation of mode fields in the basis of circular polarization, but the systematic study of optical fiber's fields concerning their dislocation structure had begun fifteen years later [16]. In a number of works by A. Volyar and T. Fadeyeva [17-19] there have been studied the dislocations and disclinations of lower mode fields in ideal round fibers. There have been demonstrated that unlike optical vortices in vacuum, guiding vortices, propagating in the inhomogeneous medium of an optical fiber, possess strict- 


\section{C.N. Alexeyev et al.: Spin-orbit interaction in a generic vortex field transmitted ...}

ly correlating values of topological charge $l$ and polarization $s$, so that for $l= \pm 1, l \sigma \neq-1$. Guided vortices meeting the requirement of this «selection rule» are conventionally called circular vortices (CV). Other vortices with $\sigma l=-1$ are called instability vortices (IV). While propagating the IV-fields, being not the eigenmodes of an ideal fiber, decompose on the TE and TM modes possessing different propagation constants $\beta_{\mathrm{TE}}$ and $\beta_{\mathrm{TM}}$, respectively [20]. It is somewhat convenient to designate the states of guiding vortices as $|\sigma, l\rangle$, so those homogeneous vortices will bear the designation $|+1,+l\rangle$ and $|-1,-l\rangle$ while inhomogeneous ones: $|+1,-l\rangle$ and $|-1,+l\rangle$. Respectively, two types of IV vortices acquire the designation $|+1,-1\rangle$ and $-1,+1$. As well as optical vortices in vacuum, guiding vortices in fibers carry the orbital and spin angular momentum [20-22]. In particular, it was demonstrated that in weakly guiding fibers with arbitrary refractive index profile the value of the angular momentum per photon coincides with that of Laguerre-Gaussian beams in a void if the propagation of light is effectively a paraxial one.

It is easily ascertained that CV with the same topological charge $l$ and opposite polarization have different propagation constants. First this fact was singled out as a manifestation of the spin-orbit interaction by B.Ya. Zel'dovich and V.S. Liberman in [23]. Further progress in the study of the spin-orbit interaction was achieved in the works $[24,25]$. There was pointed out that the spinorbit interaction plays the decisive role in the evolution of optical vortices in fibers. If we «switch abruptly off» this interaction, vortices with the same $l$ acquire the same spin-independent propagation constant $\widetilde{\beta}$. Thus, in the absence of this interaction, all the vortices in fibers, and in particular IV, are stable. Vice versa, if we "switch abrupt-ly on» the interaction, the vortices become polarization-sensitive and their propagation constant $\widetilde{\beta}$ acqui-res certain polarization corrections $\delta \beta$. It is possible to represent the value of $\delta \beta$ as an average value $\delta \beta=$ $<\psi|\delta \mathrm{H}| \psi>$, where $\delta \mathrm{H}$ is the Hamiltonian of the spinorbit interaction, first introduced in [23]. The reason for such splitting of «energy levels» lies in the fact that in the absence of interaction each eigenvalue $\widetilde{\beta}$ is four-times degenerated (except for $l=0$ that is two - time degenerated). In other words, for each 1 there exist four types of fields with different oddity and polarization which propagate with the same constant $\widetilde{\beta}$. Interaction eliminates the degeneration and splits the «levels» of $\widetilde{\beta}$.

The spin-orbit interaction is not the only existing mechanism eliminating the degeneration in fibers: the changing of a fiber cross-section can also produce such an effect. Real fibers usually have their own or externally induced defects of a cross-section. If such defects are not accompanied by the photoelastic effect, they cause basically geometric splitting of $\widetilde{\beta}$ known as «birefringence of the form» [29]. In the cross-section of elliptically deformed fibers there exist two singled out directions aligned with ellipse's axes, so the modes polarized along these directions propagate with different phase velocities. Since birefringent systems are known to trans- form the angular momentum and generate optical vortices $[7-8,26]$ there exists possibility to use elliptically deformed fibers as mode converters.

The question of electromagnetic fields in deformed fibers has been under consideration for a long time (see, for instance, the list of references, quoted in the paper [27]). In this paper there have been carried out the analysis of birefringence in single-mode fibers under various deformations, including purely geometrical ones, with the purpose of finding out the ways to obtain single-mode optical fibers not distorting linearly polarized light. The results obtained in this work enable us to calculate the polarization correction to the propagation constant of the fundamental $\mathrm{HE}_{11}$ mode for various types of deformations. However, there was suggested no regular procedure to obtain the polarization corrections to propagation constants of higher order modes. In the fundamental works by A. Snyder et al. [15,28] on the basis of a perturbation theory there have been obtained the expressions for fields and polarization corrections to propagation constants in elliptically deformed fibers (see for instance Tab. 13.1 in [15]). However, in the structure of perturbation there was included only the spin-orbit interaction, while the perturbation of the form was accounted in the zero approximation. Thus, the degeneration degree of the ground state was found to be two, what might have led to wrong conclusions at relatively small deformations of a cross-section. Moreover, the influence of the deformation on the spin-orbit interaction was also neglected.

The main objective of the present paper is to give a consistent theoretical description of optical vortices in weakly guiding elliptically deformed fibers, based on the analysis of the vector wave equation solutions, obtained by the perturbation theory method.

In Sec. 2 we consider eigenfunctions and eigenvalues of the spin - orbit interaction Hamiltonian as guided modes and their constant propagations of a weakly guiding elliptic fiber. The optical vortex behaviour in that fiber is discussed in Sec.3. In Sec. 4 we obtain the relationships between the spin and orbit angular momenta and some conclusions are drawn in Sec.5.

\section{The spin - orbit interaction, polarization corrections and eigenmodes}

It is possible to represent the vector wave equation for the transverse electric field $\mathbf{e}_{\mathrm{t}}$ in a fiber in the form:

$$
\left(\nabla^{2}+n^{2}(x, y) k^{2}\right) \mathbf{e}_{t}+\nabla_{t}\left(\mathbf{e}_{t} \nabla_{t} \ln n^{2}(x, y)\right)=\beta^{2} \mathbf{e}_{t},
$$

where $n^{2}(x, y)=n_{c o}(1-2 \Delta f(x, y)), n_{c o}$ is the refractive index along fiber's axis, $\Delta$ is the height of the refractive index profile $n^{2}(x, y), \nabla_{t} \equiv \mathbf{i} \partial / \partial x+\mathbf{j} \partial / \partial y, k=2 \pi / \lambda$ (we use the designations of the work [15]).

Consider the ideal case when the elliptic deformation of a cross-section does not induce mechanical tensions, and one can disregard with the photoelastic effect. We shall describe the ellipticity of a fiber by the parameter $\delta<<1$, which 


\section{C.N. Alexeyev et al.: Spin-orbit interaction in a generic vortex field transmitted ...}

we shall introduce as: $f(x, y) \Rightarrow f(x(1+\delta), y(1-\delta)) \approx$ $\approx \tilde{f}(\mathbf{r})+\left(\frac{\partial \tilde{f}}{\partial x} x-\frac{\partial \tilde{f}}{\partial y} y\right) \delta$, where the function $\tilde{f}(\mathbf{r})=\tilde{f}(r)$ describes the symmetric profile in a non-deformed crosssection. Note that $\delta \approx e^{2} / 4$, where $e$ stands for eccentricity of the deformation ellipse. Then the small deformation of a cross-section causes the change in $n^{2}$ and its gradient $\nabla_{t} \ln n^{2}$ in the following form:

$$
\begin{aligned}
& n^{2} \Rightarrow \widetilde{n}^{2}-2 n_{c o} \Delta \delta \cdot r^{2} \psi \cos 2 \varphi= \\
& =\widetilde{n}^{2}+\Delta n^{2} \\
& \nabla_{t} \ln n^{2} \Rightarrow-2 \Delta \nabla_{t} \tilde{f}-2 \Delta \delta\left\{\mathbf{i}\left(\tilde{f}_{x x} x+\widetilde{f}_{x}-\widetilde{f}_{x y} y\right)+\right. \\
& \left.+\mathbf{j}\left(\widetilde{f}_{x y} x-\widetilde{f}_{y y} y-\widetilde{f}_{y}\right)\right\}
\end{aligned}
$$

Here $\tilde{n}$ stands for unperturbed refractive index function $n(x, y), \psi \equiv \frac{1}{r} \frac{\partial \tilde{f}}{\partial r}, \widetilde{f}_{x y} \equiv \frac{\partial \tilde{f}}{\partial x \partial y}$, for instance. We shall use the operator approach suggested in [21,23-25] and treat the appearance of the perturbation terms $k^{2} \Delta n^{2}$ and $\nabla_{t}\left(\mathbf{e}_{t} \nabla_{t} \ln n^{2}\right)$ in (1) as a manifestation of the spinorbit coupling. Then the term $2 \Delta \nabla f$ in (2) describes the «ordinary» spin - orbit interaction, the term $\Delta n^{2}$ - the perturbation due to profile's ellipticity. The other terms, proportional to $\delta$, describe the variation of the spin-orbit interaction caused by elliptic deformation.

To simplify the analysis we shall rewrite (1) in a matrix form in the basis of circular polarization:

$$
\left(H_{0}+\hat{V}\right)|\Psi\rangle=\beta^{2}|\Psi\rangle,
$$

where $|\Psi\rangle=\frac{1}{\sqrt{2}}\left(\begin{array}{l}e_{x}-i e_{y} \\ e_{x}+i e_{y}\end{array}\right), \hat{H}_{0}=\left(\nabla^{2}+k^{2} \widetilde{n}^{2}(x, y)\right) \hat{1}$ and the expression for $\hat{V}$ is easily established from (1). Formally the equation (3) is an equation on eigenfunctions $|\Psi\rangle$ and eigenvalues $\beta^{2}$ of the Hamiltonian $\hat{H}=\hat{H}_{0}+$ $+\hat{V}$. In the absence of interaction, each eigenvalue $\beta^{2}$ is four-times degenerated: twice in polarization s and twice in orbital number 1 . The interaction eliminates the degeneration and at the same time changes the structure of the ground state. The last our case implies the choice of proper linear combinations of basic eigenfunctions belonging to the same $\beta^{2}{ }_{n, l}$. It is convenient to choose the optical vortices $[20,21]$ as such basic functions:

$\left|\Psi_{1}\right\rangle \equiv|1, l\rangle,\left|\Psi_{2}\right\rangle \equiv|1,-l\rangle,\left|\Psi_{3}\right\rangle \equiv|-1,-l\rangle,\left|\Psi_{4}\right\rangle \equiv|-1, l\rangle$,

where the first index in the vector $|$,$\rangle specifies the direc-$ tion of vortex circular polarization, $\sigma= \pm 1$, while the second - its topological charge $l$. For example, $\left\langle\sigma, l \mid \sigma^{\prime}, l^{\prime}\right\rangle=\delta_{l l^{\prime}} \delta_{\sigma \sigma^{\prime}} R=r / r_{0}$, where $F_{l}(R)$ is the ra- dial function of a state, $r_{0}$ is a core radius. One also assumes to be $\left\langle\sigma, l \mid \sigma^{\prime}, l^{\prime}\right\rangle=\delta_{l l^{\prime}} \delta_{\sigma \sigma^{\prime}}$, where

$$
\langle\Phi \mid \Psi\rangle=\iint_{S} d x d y\left(\Phi_{+}^{*}, \Phi_{-}^{*}\right)\left(\begin{array}{l}
\Psi_{+} \\
\Psi_{-}
\end{array}\right) .
$$

Note that this assumption implies the following normali-

zation of $F_{l}(R): 2 \pi \int_{0}^{\infty} R F_{l}^{2}(R) d R=1$. It is worth noting that the expressions (4) are written in the basis of circular polarization. The polarization corrections to the propagation constant $\beta^{2}{ }_{n, l}$ are found as the solutions of the characteristic equation:

$\operatorname{det}\left|V_{i j}-\delta_{i j} \beta^{2}\right|=0$.

To solve this equation one has to know the matrix elements $V_{i j}$ in the chosen basis. It is possible to prove that though $\hat{V}^{+} \neq \hat{V}$, in the basis (4) we have $\langle i|\hat{V}| j\rangle=$ $=\left\langle i\left|\hat{V}^{+}\right| j\right\rangle$. This notion enables us to use in calculations for the sake of simplicity the Hermitian Hamiltonian instead of $\hat{V}$. Then the effective Hamiltonian of the spinorbit interaction in elliptic fibers takes the form:

$$
\begin{aligned}
& \delta \hat{H}=-\Delta \psi \hat{l}_{z} \sigma_{z}-(\Delta / 2) r^{2} \chi\left(\begin{array}{cc}
0 & e^{-2 i \varphi} \\
e^{2 i \varphi} & 0
\end{array}\right)- \\
& -\Delta \psi-(\Delta / 2) r^{2} \chi-\Delta \delta\left\{\left[2 n_{c o} r^{2} r^{2} \psi k^{2}+\frac{\left(r^{6} \chi\right)^{\prime}}{2 r^{3}}\right] \cos 2 \varphi \cdot \hat{1}+\right. \\
& \left.+\left(2 \widetilde{f}^{\prime \prime}+\frac{1}{4} r^{3} \chi^{\prime}\right) \sigma_{x}+\frac{1}{4} r^{3} \chi\left(\begin{array}{cc}
0 & e^{-4 i \varphi} \\
e^{4 i \varphi} & 0
\end{array}\right)\right\}
\end{aligned}
$$

where $\chi \equiv \frac{1}{r} \frac{\partial \psi}{\partial r}, \hat{l}_{z}=-i \frac{\partial}{\partial \varphi}$ is the operator of the $z$ component of the angular momentum, $\hat{1}, \sigma_{x, z}$ are Pauli matrices. In (6) we have omitted two terms which give zero contribution to matrix elements. The first four terms form the Hamiltonian of the spin-orbit interaction in round fibers [21,23-25].

For various $l$ the matrix $\delta H_{i j}$ has the following structure, determined by the symmetry of interaction:

$$
\delta \hat{H}=\left(\begin{array}{cccc}
A_{l} & D_{l} & C_{l} & E_{l} \\
D_{l} & B_{l} & E_{l} & C_{l}+G_{l} \\
C_{l} & E_{l} & A_{l} & D_{l} \\
E_{l} & C_{l}+G_{l} & D_{l} & B_{l}
\end{array}\right) .
$$




\section{C.N. Alexeyev et al.: Spin-orbit interaction in a generic vortex field transmitted ...}

We shall obtain the explicit expressions for eigenvectors of this matrix and the spectrum of corrections to the eigenvalues of the operator $\hat{H}_{0}$ for fibers with step and parabolic refractive index profiles. The function $f$ of the step refractive index profile is given by $f(R)=\left\{\begin{array}{l}0, R \leq 1 \\ 1, R>1\end{array}\right.$, while for the truncated parabolic profile it is $f(R)=\left\{\begin{array}{c}R^{2}, R \leq 1 \\ 1, R>1\end{array}\right.$.

1. The fundamental mode, $l=0$. From (6) we obtain: $A_{0}=B_{0}, D_{0}=0, G_{0}=0, E_{0}=C_{0}$. Then for eigenvectors we have: $\left|e_{1}\right\rangle=|1,0\rangle-|-1,0\rangle,\left|e_{4}\right\rangle=|1,0\rangle+|-1,0\rangle$, while for polarization corrections: $\Delta \beta_{1}^{2}=A_{0}+2 C_{0}$, $\Delta \beta_{4}{ }^{2}=A_{0}-2 C_{0}$. The explicit expression of the matrix elements is given in the Table 2 . The result obtained demonstrates the absence of degeneration. Note that we have not normalized the eigenvectors.

2. The lowest order mode, $l=1$. In this case $C_{1}=0$ and the degeneration is also completely eliminated. The eigenvectors given in the Table 1 by their structure present certain combinations of modes in round weakly guiding fibers. In the designations of [15] one can represent the modes of elliptical weakly guiding fibers also as:

$$
\begin{aligned}
& \left|\Psi_{1}\right\rangle=i\left(K_{1}\left|H E^{o d} l+1, m\right\rangle-\left|E H^{o d} l-1, m\right\rangle\right), \\
& \left|\Psi_{2}\right\rangle=i\left(K_{2}\left|H E^{o d} l+1, m\right\rangle-\left|E H^{o d} l-1, m\right\rangle\right), \\
& \left|\Psi_{3}\right\rangle=-K_{3}\left|H E^{e v} l+1, m\right\rangle+\left|E H^{e v} l-1, m\right\rangle, \\
& \left|\Psi_{4}\right\rangle=-K_{4}\left|H E^{e v} l+1, m\right\rangle+\left|E H^{e v} l-1, m\right\rangle,
\end{aligned}
$$

where $K_{i}$ are defined in the Table 1, HE and EH-symbols stand for the standard designations of modes in ideal fibers, the upper indices ev and od specify mode's oddity, $\mathrm{m}$ is the radial index. It is easily demonstrated that the obtained expressions (8) for mode fields can be also written as:

$$
\begin{aligned}
& \left|\Psi_{1,2}\right\rangle=\left\{\hat{\mathbf{x}} \sin (l \varphi)+\hat{\mathbf{y}}\left(\Lambda_{1} \pm \sqrt{1+\Lambda_{1}^{2}}\right) \cos (l \varphi)\right\} F_{l}(R), \\
& \left|\Psi_{3,4}\right\rangle=\left\{\hat{\mathbf{x}} \cos (l \varphi)+\hat{\mathbf{y}}\left(\Lambda_{2} \mp \sqrt{1+\Lambda_{2}^{2}}\right) \sin (l \varphi)\right\} F_{l}(R),
\end{aligned}
$$

where $\mathrm{x}, \mathrm{y}$ are the unit vectors, $F_{l}(R)$ is the radial function, $\Lambda_{1,2}=\frac{D_{l} \mp E_{l}}{A_{l}-B_{l} \pm G_{l}}$. The comparison with the re-

Table 1. Eigenvectors and polarization corrections to a propagation constant in an elliptic fiber

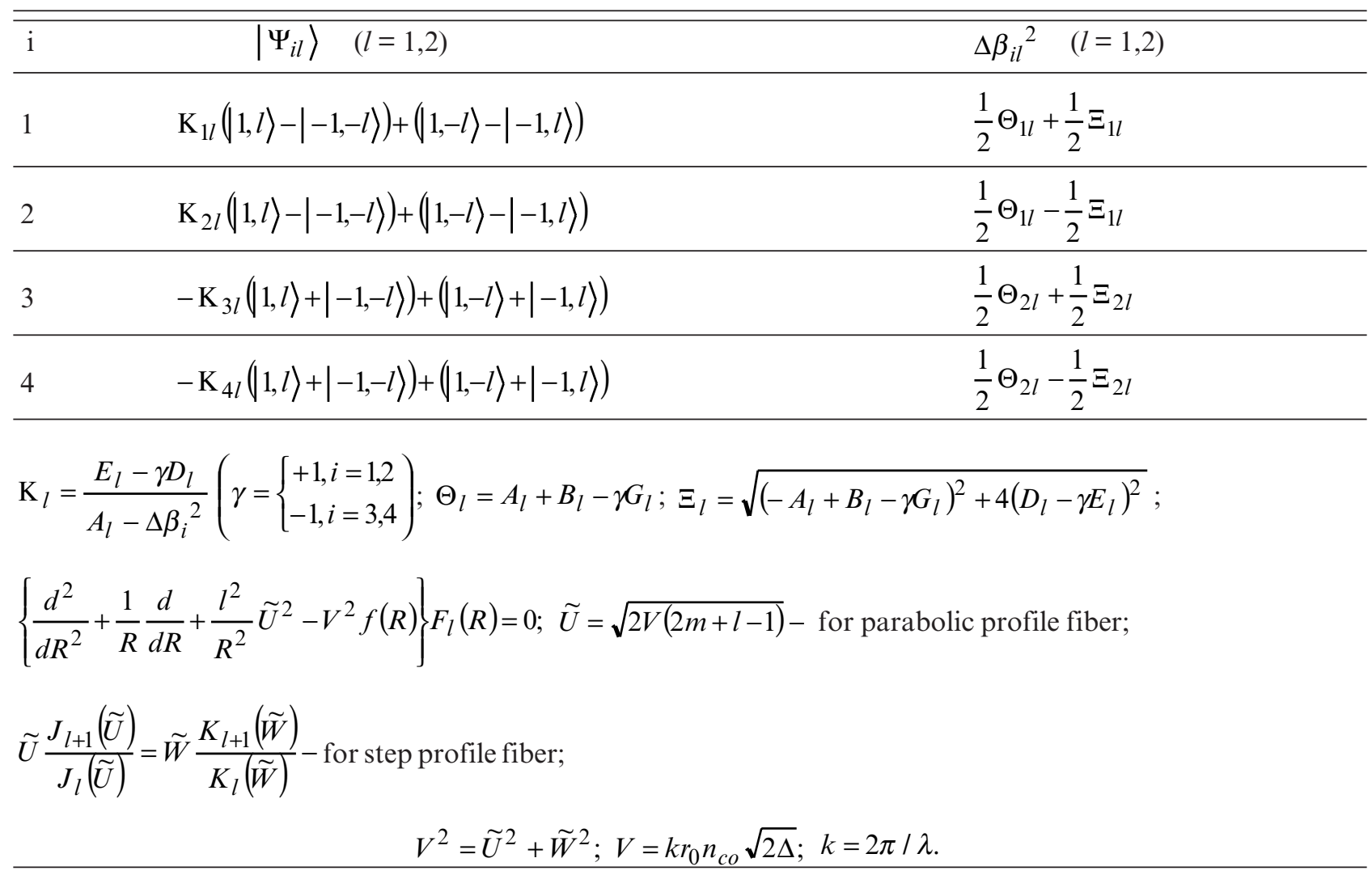


C.N. Alexeyev et al.: Spin-orbit interaction in a generic vortex field transmitted ...

Table 2. Matrix elements of the spin-orbit interaction operator $\delta \hat{H}$ in an elliptic fiber

\begin{tabular}{|c|c|}
\hline Step index profile & Parabolic index profile \\
\hline$l=0$ & $l=0$ \\
\hline$A_{0}=-\left.\frac{\Delta}{r_{0}^{2}} F_{0} F_{0}\right|_{R=1}$ & $A_{0}=\frac{\Delta}{r_{0}^{2}}\left\{\left.F_{0}^{2}\right|_{R=1}-2 \int_{0}^{1} R F_{0}^{2} d R\right\}$ \\
\hline$C_{0}=-\frac{\delta \Delta}{2 r_{0}^{2}}\left(F_{0} F_{0}^{\prime \prime}+F_{o}^{\prime 2}-F_{0} F_{0}^{\prime}\right) R=1$. & $C_{0}=\frac{\delta \Delta}{r_{0}^{2}}\left\{\left(2 F_{0}^{2}-F_{0} F_{0}{ }^{\prime}\right)_{R=1} \cdot-4 \int_{0}^{1} R F_{0}^{2} d R\right\}$. \\
\hline$l=1,2$ & $l=1,2$ \\
\hline$A_{l}=-\frac{\Delta}{r_{0}^{2}}\left(F_{l}^{2}-F_{l} F_{l}^{\prime}\right)_{R=1}$ & $A_{l}=-\frac{4 \Delta}{r_{0}^{2}}\left\{\int_{0}^{1} R F_{l}^{2} d R-\left.\frac{1}{4} F_{l}^{2}\right|_{R=1}\right\}$ \\
\hline$B_{l}=\frac{\Delta}{r_{0}^{2}}\left(F_{l}^{2}+F_{l} F_{l}^{\prime}\right)_{R=1} ; C_{l}=0$ & $B_{l}=-\left.\frac{\Delta}{r_{0}^{2}} F_{l}^{2}\right|_{R=1} ; C_{l}=0$ \\
\hline$D_{l}=\frac{1}{2} k^{2} n_{c o}^{2} \delta^{2} \Delta\left(a_{l} F_{l}^{2}+b_{l} F_{l} F_{l}^{\prime}\right)_{R=1}$ & $D_{l}=-2 \delta^{2} \Delta k^{2} n_{c o}{ }^{2}\left\{\varepsilon_{l} \int_{0}^{1} R^{4-l} F_{l}^{2} d R+\left.\frac{b_{l}}{4} F_{l}^{2}\right|_{R=1}\right.$ \\
\hline$E_{l}=-\frac{\delta \Delta}{2 r_{0}^{2}}\left(F_{l} F_{l}^{\prime \prime}+F_{l}^{\prime 2}-F_{l} F_{l}^{\prime}\right)_{R=1}$ & $E_{l}=\frac{\delta \Delta}{r_{0}^{2}}\left\{\left(2 F_{l}^{2}-F_{l}^{\prime} F_{l}\right)_{R=1}-4 \int_{0}^{1} R F_{l}^{2} d R\right\}$ \\
\hline$G_{l}=\frac{\delta \Delta}{r_{0}^{2}}\left\{p_{l} F_{l}^{2}+d_{l} F_{l} F_{l}^{\prime}-2 b_{l}\left(F_{l}^{2}+F_{l} F_{l}{ }^{\prime \prime}\right)\right\}_{R=1}$ & $G_{l}=-\frac{4 \delta \Delta}{r_{0}^{2}}\left(\kappa_{l} F_{l}^{2}+b_{l} F_{l} F_{l}^{\prime}\right)_{R=1}$ \\
\hline
\end{tabular}

$$
a_{l}=\left\{\begin{array}{l}
-\frac{2}{\delta}, l=1 \\
\frac{5}{2}, l=2
\end{array} ; b_{l}=\left\{\begin{array}{l}
0, l=1 \\
1, l=2
\end{array} ; d_{l}=\left\{\begin{array}{c}
\frac{1}{\delta}, l=1 \\
-14, l=2
\end{array} ; p_{l}=\left\{\begin{array}{c}
\frac{1}{\delta}, l=1 \\
-8, l=2
\end{array} ; \varepsilon_{l}=\left\{\begin{array}{l}
\frac{1}{\delta}, l=1 \\
-\frac{1}{2}, l=2
\end{array} ; \kappa_{l}=\left\{\begin{array}{l}
\frac{1}{4 \delta}, l=1 \\
2, l=2
\end{array}\right.\right.\right.\right.\right.\right.
$$

sults of [15] reveals an agreement with the form of notation at $E_{l}=0$. Though this definition of $\Lambda$ differs from the one given in [15], essentially $\Lambda$ is the ratio of ellip-ticityinduced splitting to the splitting caused by pure spin-orbit interaction, what is also in agreement with [15].

Consider some limiting cases, namely: 1 ) the case of small ellipticity with $\delta<<\left(\lambda / r_{0}\right)^{2}$ and 2) the case of large ellipticity with $\left(\lambda / r_{0}\right)^{2}<<\delta<<1$. The margin $\delta_{0} \equiv$ $\equiv\left(\lambda / r_{0}\right)^{2}$ is found as the $\delta$ at which the contribution of purely spin-orbit terms which are of order $\Delta / r_{0}^{2}$ to polarization corrections is comparable with that of purely ellipticity-induced terms which we find proportional to $\delta \Delta / \lambda^{2}$.

A) Small ellipticity: $\delta<<\delta_{0}$. From the Tables 1 and 2 we readily obtain:

$$
\begin{aligned}
& K_{1} \approx-D_{1} /\left\{0.5\left(A_{1}-\left|A_{1}\right|\right)-D_{1}^{2} /\left|A_{1}\right|\right\}, \\
& K_{2} \approx-D_{1} /\left\{0.5\left(A_{1}+\left|A_{1}\right|\right)+D_{1}^{2} /\left|A_{1}\right|\right\}, \\
& K_{3} \approx-D_{1} /\left\{0.5\left(A_{1}-2 B_{1}-\left|A_{1}-2 B_{1}\right|\right)-D_{1}^{2} /\left|A_{1}-2 B_{1}\right|\right\}, \\
& K_{4} \approx-D_{1} /\left\{0.5\left(A_{1}-2 B_{1}+\left|A_{1}-2 B_{1}\right|\right)+D_{1}^{2} /\left|A_{1}-2 B_{1}\right|\right\} .
\end{aligned}
$$

For very small ellipticity at certain assumptions on the signs of $A_{1}$ and $A_{1}-2 B_{1}$ from (8) we obtain: $\left|\Psi_{1}\right\rangle \approx$ $\approx-i\left|H E^{o d}{ }_{21}\right\rangle,\left|\Psi_{3}\right\rangle \approx-\left|H E^{e v}\right\rangle,\left|\Psi_{2}\right\rangle \approx i\left|T E_{0, m}\right\rangle$, 


\section{C.N. Alexeyev et al.: Spin-orbit interaction in a generic vortex field transmitted ...}

$\left|\Psi_{4}\right\rangle \approx\left|T M_{0, m}\right\rangle$, so that in the limit $\delta \rightarrow 0$ we come to the modes of an ideal fiber. Note that the other choice of signs would lead to a mere renumbering of the states. Though for small ellipticity we can disregard with its influence on mode amplitudes, in the polarization corrections we cannot neglect it. So from the Tables 1 and 2 for the polarization corrections we obtain:

$$
\begin{aligned}
& \Delta{\beta_{1}}^{2} \approx 0.5\left(A_{1}+\left|A_{1}\right|\right)+D_{1}^{2} /\left|A_{1}\right|, \\
& \Delta \beta_{2}{ }^{2} \approx 0.5\left(A_{1}-\left|A_{1}\right|\right)-D_{1}{ }^{2}|| A_{1} \mid, \\
& \Delta \beta_{3}{ }^{2} \approx 0.5\left(A_{1}+2 B_{1}+\left|A_{1}-2 B_{1}\right|\right)+D_{1}{ }^{2}|| A_{1}-2 B_{1} \mid, \\
& \Delta \beta_{4}{ }^{2} \approx 0.5\left(A_{1}+2 B_{1}-\left|A_{1}-2 B_{1}\right|\right)-D_{1}{ }^{2} /\left|A_{1}-2 B_{1}\right| .
\end{aligned}
$$

In the limit $\delta \rightarrow 0$ the corrections (11) coincide with the polarization corrections to the propagation constant $\widetilde{\beta}$ given in Tables 14.1 and 14.2 of [15]. It should be stressed that the splitting of «energy levels» $\beta_{n, l}$ is of order $\delta^{2}$. Since $\delta \propto e^{2}$, where $e$ is the eccentricity, then $\Delta \beta \propto e^{4}$ what contradicts to the result that could have been obtained on the basis of expressions derived in [15] $\left(\Delta \beta \propto e^{2}\right)$. The reason of such discordance lies in the difference in the choice of the zeroth-approximation Hamiltonian and therefore the structure of perturbation in the eq. (1).

B) Large ellipticity: $\delta_{0}<<\delta<1$. In this case from the Tables 1 and 2 for $K_{i}$ we obtain: $\left|K_{i}\right| \approx 1$. In the basis of linear polarization the expressions (8) for the fields can be recast as:

$$
\begin{aligned}
\left|\Psi_{1}\right\rangle & \left.\left.\propto\left(\begin{array}{c}
\sin \varphi \\
0
\end{array}\right), \Psi_{2}\right\rangle \propto\left(\begin{array}{c}
0 \\
\cos \varphi
\end{array}\right) \Psi_{3}\right\rangle \propto\left(\begin{array}{c}
0 \\
\sin \varphi
\end{array}\right) \\
\Psi_{4} & \propto\left(\begin{array}{c}
\cos \varphi \\
0
\end{array}\right)
\end{aligned}
$$

So that this case is reduced to the well studied case of LP-modes [15]. The polarization corrections to $\widetilde{\beta}$ are given by:

$$
\begin{aligned}
& \Delta \beta_{1}{ }^{2} \approx\left|D_{1}\right|+0.5 A_{1}, \Delta \beta_{2}{ }^{2} \approx\left|D_{1}\right|-0.5 A_{1}, \\
& \Delta \beta_{3}{ }^{2} \approx 0.5 A_{1}+B_{1}+\left|D_{1}\right|, \Delta \beta_{4}{ }^{2} \approx 0.5 A_{1}+B_{1}-\left|D_{1}\right| .
\end{aligned}
$$

Note that the splitting of «levels» is of order $\delta$, or $e^{2}$, what is in agreement with the corresponding result of [15].

Before proceeding to the optical vortices behaviour, it is worth to discuss briefly the limits of the perturbation theory applicability.

In general, the perturbation theory is valid if the corrections to $\widetilde{\beta}$ are much less then the difference between the neighboring levels $\widetilde{\beta}_{n, l}: \Delta \beta<\left|\widetilde{\beta}_{n}-\widetilde{\beta}_{m}\right|$ [32]. At relatively large values of the deformation parameter $\delta$ $(l=1)$ we have:
$\Delta \beta^{2} \propto D \propto \frac{\Delta \delta}{\lambda^{2}} n^{2} \Rightarrow \Delta \beta \propto \frac{\Delta \beta^{2}}{\widetilde{\beta}} \propto \frac{\Delta \beta n^{2}}{\lambda^{2} \widetilde{\beta}}$.

On the other hand, in weakly guiding fibers $[15]: \mid \widetilde{\beta}_{n}-$ $-\widetilde{\beta}_{m} \propto k\left(n_{c o}-n_{c l}\right) \propto k n \Delta$, so that one can express the condition of the perturbation theory applicability as:

$\Delta \beta<\left|\widetilde{\beta}_{n}-\widetilde{\beta}_{m}\right|, \frac{\Delta \delta n^{2}}{\lambda^{2} \widetilde{\beta}} \ll<n k \Delta \Rightarrow \delta<<1$.

In calculations we usually use the correction $\delta \beta_{i}$ to the propagation constant $\widetilde{\beta}$ rather than the correction $\Delta \beta^{2}$ to the eigenvalue $\widetilde{\beta}^{2}$ of the operator $\hat{H}_{0}$. Determine the connection between these two corrections. As follows from the definition, the eigenvalues of the perturbed Hamiltonian $\hat{H}=\hat{H}_{0}+\delta \hat{H}$ are $\beta_{i}^{2}=\widetilde{\beta}^{2}+\Delta \beta_{i}^{2}$, where $i$ is the number of a level in the quadruplet. Then $\beta_{i}=\sqrt{\widetilde{\beta}^{2}+\Delta \beta_{i}^{2}} \approx \widetilde{\beta}+\delta \beta_{i}$, where $\delta \beta_{i}=\frac{\Delta \beta_{i}^{2}}{2 \widetilde{\beta}}$.

\section{Generic optical vortices, their propagation and conversion}

As evident, the optical vortex being not the eigenmode of an elliptic fiber cannot propagate in it without any changes. If the vortex is launched into a slightly elliptic fiber, it generally decomposes into all the four types of vortices $|\sigma, l\rangle$ and they become the generic vortices:

$|C V\rangle^{e l l}=\sum_{j} C_{j}\left|\Psi_{j}\right\rangle e^{i \beta_{j} z}$

where $\left|\Psi_{j}\right\rangle$ is the $j$-th eigenmode of an ideal fiber, $C_{j}$ is a weight coefficient. If the fiber is excited with the vortex

$\sigma, l$ then on its input $(z=0)$ we have the boundary condition:

$|1, l\rangle=\sum_{j} C_{j}\left|\Psi_{j}\right\rangle$,

which provides the system of equations for coefficients $C_{j}$.

\subsection{Homogeneous generic vortices $| \pm 1, \pm l\rangle$}

1. Small ellipticity $\left(\delta<<\delta_{0}\right), l=1$. Neglecting in field amplitudes the members small in $\left(\delta<<\delta_{0}\right)$, from the Table 2 through (15) we obtain:

$|1, l\rangle^{e l l}=\left\{|1, l\rangle \cos \delta \beta_{24} z+i|-1,-l\rangle \times\right.$ $\left.\times \sin \delta \beta_{24} z\right\} \exp \left\{i\left(\widetilde{\beta}+\beta_{24}\right) z\right\}$ 


\section{C.N. Alexeyev et al.: Spin-orbit interaction in a generic vortex field transmitted ...}

where $\delta \beta_{24}=\left(\delta \beta_{2}-\delta \beta_{4}\right) / 2, \beta_{24}=\left(\delta \beta_{2}+\delta \beta_{4}\right) / 2$. As evident, the ellipticity causes oscillations between $|1, . l\rangle$ and $|-1,-l\rangle$ vortices with the beating length $\Lambda=2 \pi / \delta \beta_{24}$. On intermediate lengths the field does not possess the definite topological charge and polarization. To analyse the polarization of the field consider Stokes parameters [29]:

$$
\begin{aligned}
& S_{0}=e_{x} e_{x}^{*}+e_{y} e_{y}^{*}=1, \\
& S_{1}=e_{x} e_{x}^{*}-e_{y} e_{y}^{*}=\sin 2 \varphi \sin 2 \delta \beta_{24} z, \\
& S_{2}=e_{x} e_{y}^{*}+e_{x}^{*} e_{y}=-\cos 2 \varphi \sin 2 \delta \beta_{24} z, \\
& S_{3}=e_{x}^{*} e_{y}-e_{x} e_{y}^{*}=\cos 2 \delta \beta_{24} z .
\end{aligned}
$$

As follows from (17) in each point the field is completely polarized: $S_{0}^{2} \equiv S_{1}^{2}+S_{2}^{2}+S_{3}^{2}$. Besides, on any radial line $\varphi=$ const polarization's ellipticity is constant while an azimuth $\psi$ of polarization ellipse changes as $\psi=\frac{\pi}{4}+\varphi$. The distribution of polarization in a transversal cross-section is given in Fig. 1.

Determine the $z$-component of the total angular momentum. In a weakly guiding fiber cross-section, the total angular momentum is given by $[21,23]$ :

$$
M_{z} \approx m_{0} \sum_{j}\left(l_{j}+\sigma_{j}\right)\left|C_{j}\right|^{2}
$$

where $m_{0}=\frac{\varepsilon \mu}{2 c^{2}} \frac{\sqrt{2 \Delta}}{V} \sqrt{\frac{\varepsilon_{0}}{\mu_{0}}} n_{c o} r_{0}^{3}$. From (18) and (16) one can obtain the linear density of the angular momentum in an elliptic fiber in the form:

$M_{z}{ }^{e l l} \approx 2 m_{0} \cos \delta \beta_{24} z$

As evident, the angular momentum oscillates and the beating length equals $2 \Lambda$, so that an elliptic fiber transforms the angular momentum as well as mode converters $[7,26]$. But unlike in mode converters, in almost round elliptic fibers the transformation of the angular momentum is not accompanied by the changes in the intensity distribution in a cross-section. As follows from (11) the beating length is:

$$
\Lambda=2 \pi \frac{\widetilde{\beta}}{D_{1}^{2}}\left(\frac{1}{\left|A_{1}-2 B_{1}\right|}-\frac{1}{\left|A_{1}\right|}\right)^{-1}
$$

so that $\Lambda \propto e^{-4}$.

2. Large ellipticity, $l=1\left(\delta>>\delta_{0}\right)$.

For definiteness we shall take $D_{1}>0$. In this case from (14) through (15) we obtain:
$1, l\rangle^{e l l}=\frac{1}{4}\left\{1, l\left(e^{i \beta_{1} z}+e^{i \beta_{2} z}+e^{i \beta_{3} z}+e^{i \beta_{4} z}\right)+\right.$

$+|-1,-l\rangle\left(-e^{i \beta_{1} z}-e^{i \beta_{2} z}+e^{i \beta_{3} z}+e^{i \beta_{4} z}\right)+$

$+|1,-l\rangle\left(e^{i \beta_{1} z}-e^{i \beta_{2} z}+e^{i \beta_{3} z}-e^{i \beta_{4} z}\right)+$

$\left.+|-1, l\rangle\left(-e^{i \beta_{1} z}+e^{i \beta_{2} z}+e^{i \beta_{3} z}-e^{i \beta_{4} z}\right)\right\}$

or for the Cartesian components of the field:

$e_{x}=\frac{1}{\sqrt{2}}\left\{\cos l \varphi \cdot e^{1 \delta \beta_{3} z}+i \sin l \varphi \cdot e^{i \delta \beta_{2} z}\right\} e^{i \widetilde{\beta} z} F_{l}(R)$
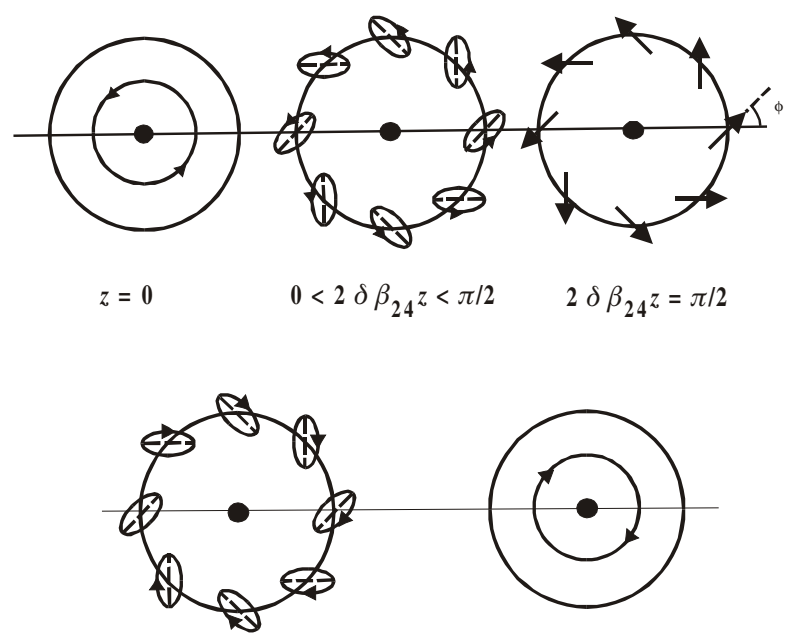

$$
\pi / 2<2 \delta \beta_{24} \mathrm{z}<\pi \quad 2 \delta \beta_{24} \mathrm{z}=\pi
$$

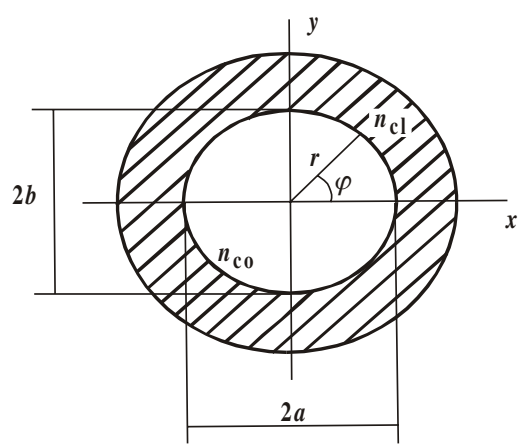

Fig. 1. The sketch representation of the polarization state distribution $S_{3}$ in several elliptical fiber cross - sections along half beating length in a small elipticity case. As a generic vortex is transmitted through a fiber, there is appeared the polarization state conversion from the right $(\sigma=+1)$ to the left $(\sigma=-1)$ circularity at the half beating length, but the cross - section intensity distribution is not altered. At an arbitrary fiber cross -section, there takes place a nonuniform polarization distribution. Nevertheless, at the each cross - section there are a set of radial senses along which the polarization state $S_{3}$ is constant. The going around of a fiber axis induces the full turn of the polarization azimuth $\phi=$ $\arctan S_{2} / S_{1}$. At the fiber axis $\operatorname{Re} \Psi=\operatorname{Im} \Psi=0$. The optical fiber parameters are $V=3.5, e=0.01, n_{c o}=1.48, \rho=4.5 \mathrm{~m} \mu$.

$S Q O, 3(4), 2000$ 


\section{C.N. Alexeyev et al.: Spin-orbit interaction in a generic vortex field transmitted ...}

$e_{y}=\frac{-i}{\sqrt{2}}\left\{\cos l \varphi \cdot e^{i \delta \beta_{1} z}+i \sin l \varphi \cdot e^{i \delta \beta_{4} z}\right\} e^{i \beta z} F_{l}(R)$.

As obvious, in this case the process of field transformation is characterised by the two beating lengths: $\Lambda_{1}$ and $\Lambda_{2}, \Lambda_{1} \gg \Lambda_{2}$. It also follows from the expression for the total angular momentum:

$M_{z} \approx 2 m_{0} \cos ^{2}\left(\frac{D_{1}}{2 \widetilde{\beta}} z\right) \cos \left(\frac{B_{1}}{2 \widetilde{\beta}} z\right)$,

(the dependence curves is given by Fig.2).

\subsection{Inhomogeneous generic vortices $| \pm 1, \mp l\rangle$}

1. Small ellipticity, $(l=1)$. With the same assumptions on the signs of $A_{1}$ and $A_{1}-2 B_{1}$ from (14), (15) we obtain:

$$
\begin{aligned}
& |1,-l\rangle^{e l l}=\left\{|1,-l\rangle \cos \delta \beta_{13} z+i|-1, l\rangle \times\right. \\
& \left.\times \sin \delta \beta_{13} z\right\} \exp \left\{i\left(\widetilde{\beta}+\beta_{13} z\right)\right\}
\end{aligned}
$$

a)

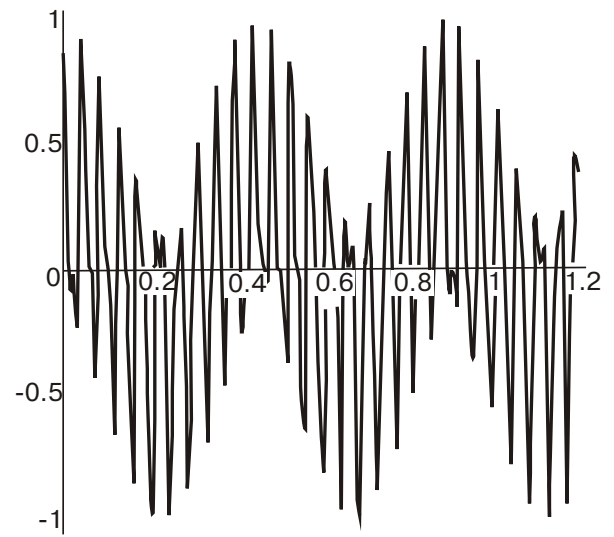

c)

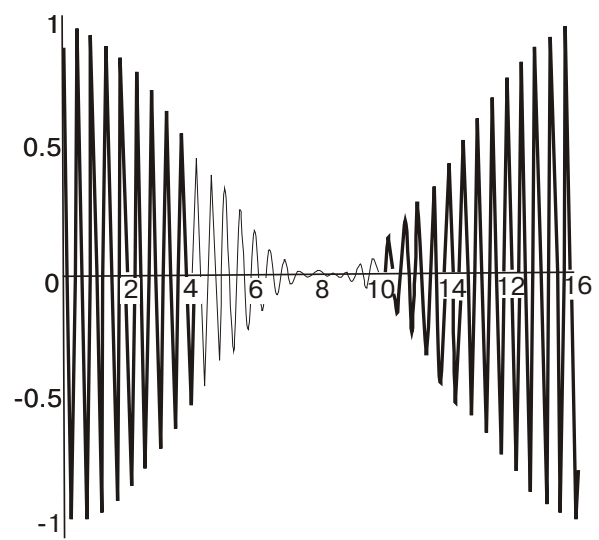

The expressions for Stokes's parameters of this field are easily obtained from (20) by the substitution: $S_{1,3} \rightarrow-S_{1,3}$. The beating length is $\Lambda=2 \pi / \delta \beta_{13}$. This field carries zero total angular momentum since the orbital and spin angular momentum have different signs and equal moduli at any $z$.

2. Large ellipticity $(l=1)$.

The transverse electric field can be written as:

$|1,-l\rangle^{e l l}=\frac{1}{4}\left\{|1, l\rangle\left(e^{i \beta_{1} z}-e^{i \beta_{2} z}+e^{i \beta_{3} z}-e^{i \beta_{4} z}\right)_{+}\right.$

$+|-1,-l\rangle\left(-e^{i \beta_{1} z}+e^{i \beta_{2} z}+e^{i \beta_{3} z}-e^{i \beta_{4} z}\right)+$

$+|1,-l\rangle\left(e^{i \beta_{1} z}+e^{i \beta_{2} z}+e^{i \beta_{3} z}+e^{i \beta_{4} z}\right)+$

$+|-1, l\rangle\left(-e^{i \beta_{1} z}-e^{i \beta_{2} z}+e^{i \beta_{3} z}+e^{i \beta_{4} z}\right)$.

As well as in the case of homogeneous vortices, the propagation of the inhomogeneous vortices $| \pm 1, \mp l\rangle$ exhibits the existence of two beating lengths: $\Lambda_{1}$ and $\Lambda_{2}$, $\Lambda_{1}>\Lambda_{2}$. The pattern of field transformation and polarization states of the field described by (25) is schematically given in Fig.3. Since the ratio $\Lambda_{1} / \Lambda_{2}$ generally exhib-

b)

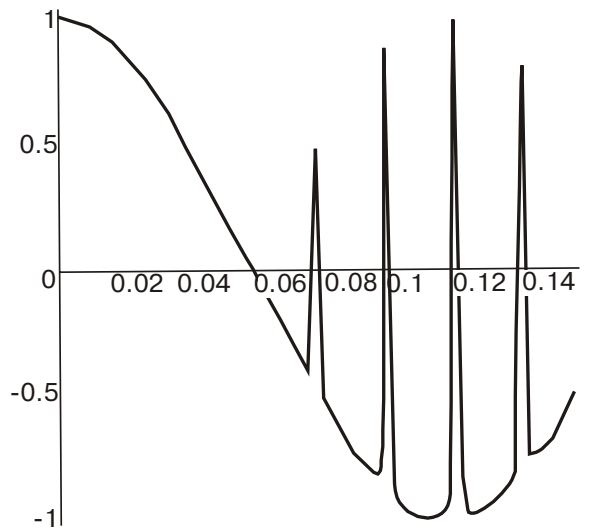

d)

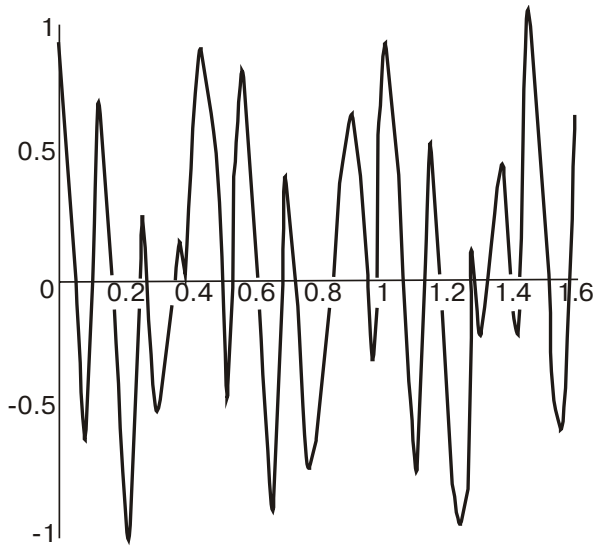

Fig. 2. The dependencies of the angular momentum $M_{z}$ and the polarization state $S_{3}$ (at the point vicinity $x=y=0.2 \mu \mathrm{m}$ ) at the fiber length $z$ (in meters) of a homogeneous vortex in the wave state $|+1,+1\rangle$ for (a-b) $e=0.2, V=3.5$ and (c-d) $e=0.01, V=3.5$ in a large ellipticity case. 


\section{C.N. Alexeyev et al.: Spin-orbit interaction in a generic vortex field transmitted ...}

its the property of irrational numbers to refine chaotically its value with the increase of accuracy, the field distribution never restores its initial pattern in the propagation process. Thus the optical vortices in highly elliptic fibers display the elements of a random behavior what is intrinsic to all physical systems with two or more characteristic oscillation parameters.

While the presence of the length $\Lambda_{2}$ corresponds to small-scale oscillations, the length $\Lambda_{1}$ governs large-scale variations of fields and exhibit itself only in fibers several meters long. So if near the input end of a fiber purely screw dislocation is transformed into a mixed dislocation, at the length $\Lambda_{1} / 4$ one can observe degenerated edge dislocation with the axis inclined at an angle $\pi / 4$. At the length $\Lambda_{1} / 2$ the degenerated edge dislocation is once more reproduced. The angular momentum changes with $z$ as:

$$
M_{z} \approx 2 m_{0} \sin ^{2}\left(\frac{D_{1}}{2 \widetilde{\beta}} z\right) \cos \left(\frac{B_{1}}{2 \widetilde{\beta}} z\right) \text {, }
$$

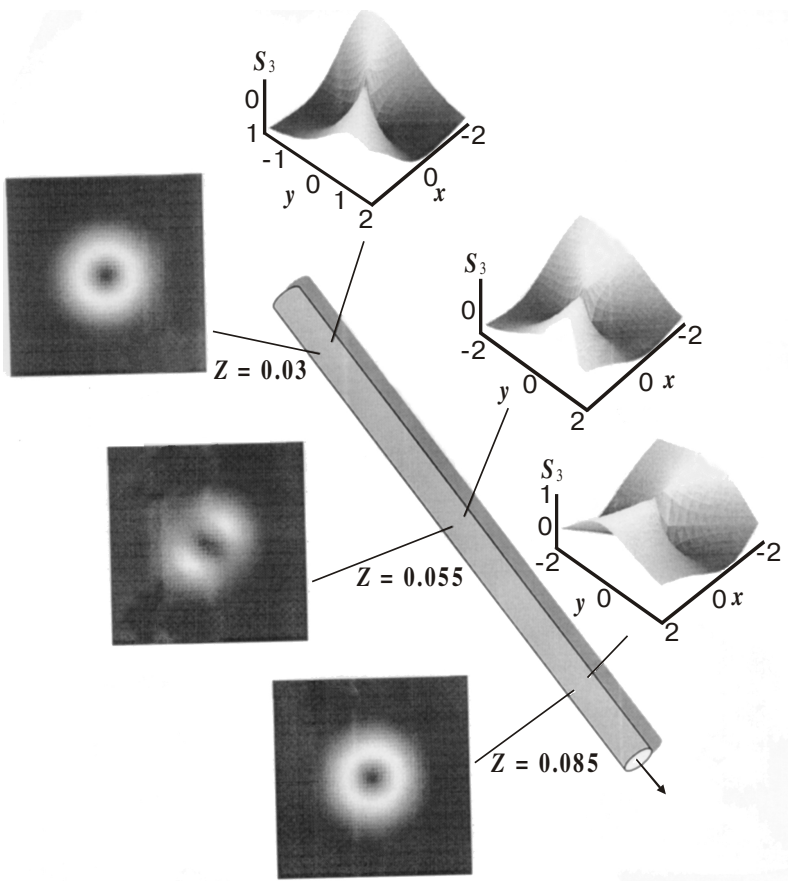

Fig. 3a. The sketch of the mode conversion of an elliptic step index optical fiber: $V=3.5, e=0.1, n_{c o}=1.48, \rho=4.5 \mathrm{~m} \mu$ at small lengths in a large elliptic case. Observed are the slight swingings of the cross - section intensity distributions $S_{0}\left(x, y, z_{0}\right)$ (from the left) due to a variation of the ellipticity degree $S_{3}\left(x, y, z_{0}\right)$ in a fiber cross - section $z_{0}$ (on the right) on the generic vortex spread. this $z$-dependence curve is given by Fig. 4 . The most distinguishing feature of this result (as well as for homogeneous vortices in highly elliptic fibers ) is the conversion of the angular momentum characterized by two lengths: $\Lambda_{1}$ and $\Lambda_{2}$. To understand the cause of that, we have to analyze the evolution of the spin and the orbital angular momentum with $z$.

\section{The spin and orbital angular momenta}

The problem of the angular momentum transformations is one of the best illustrations of the guided vortices transmitting along a perturbed fiber. It is the problem that are the base one on this process propagation, and it is the intrinsic process of the orbital and spin momenta conversion that turns vortex fields into the generic wave fields of guiding inhomogeneous media. It notes that there are no the eigen vortices in a real fiber since even a slight perturbation of a fiber decomposes those into a sum of the eigen modes that is it transforms the fields into the generic vor-

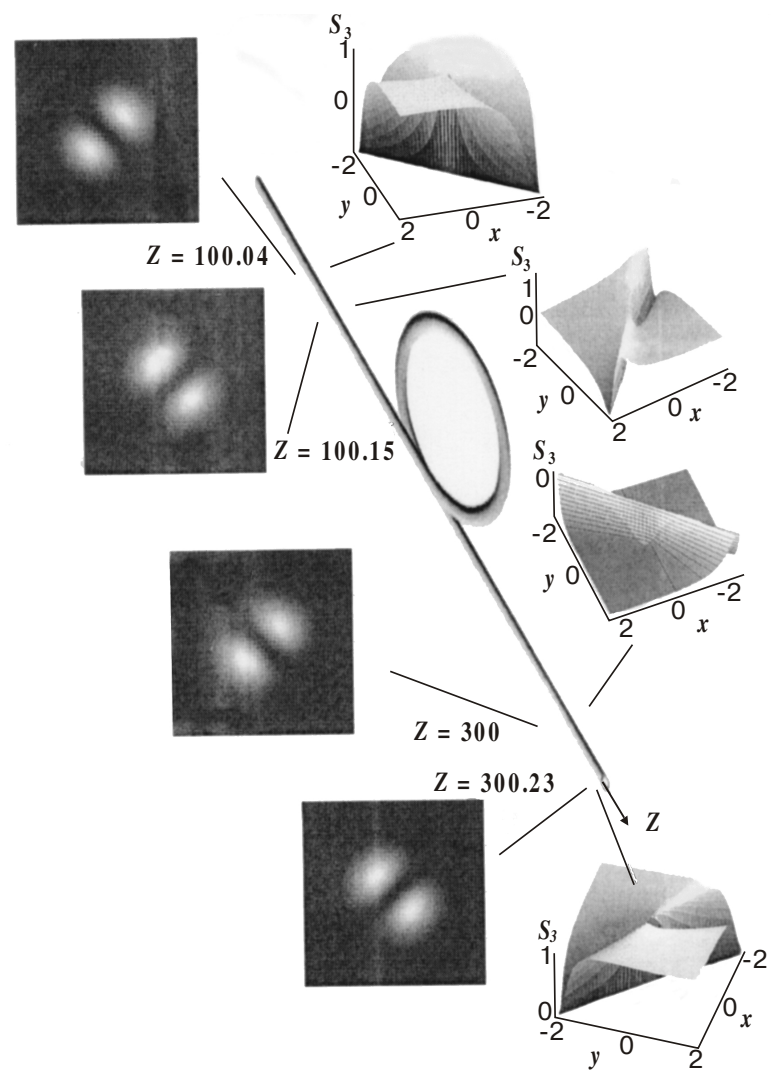

Fig. 3b. The similar situation that is the Fig. 3a but only for the lardge fiber lengths. It is observed a strong swimings both the intensity distribution $S_{0}\left(x, y, z_{0}\right)$ (from the left) and the ellipticity degree $S_{3}\left(x, y, z_{0}\right)$ (on the right). There arises the state converstion from a degenarated edge dislocation to a pure screw dislocation and from the right polarizable ellips to the left one. It notes that in the pure states, the dislocation axis and the polarizable azimuth senses are always turn out with respect to the the intial states. And there are not fiber cross - section at which the initial states could be recovered whatever the fiber length is. 
a)

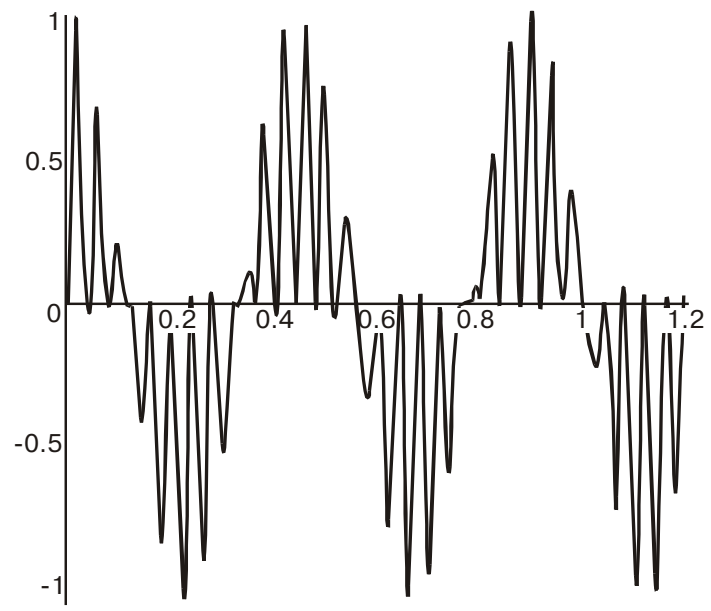

c)

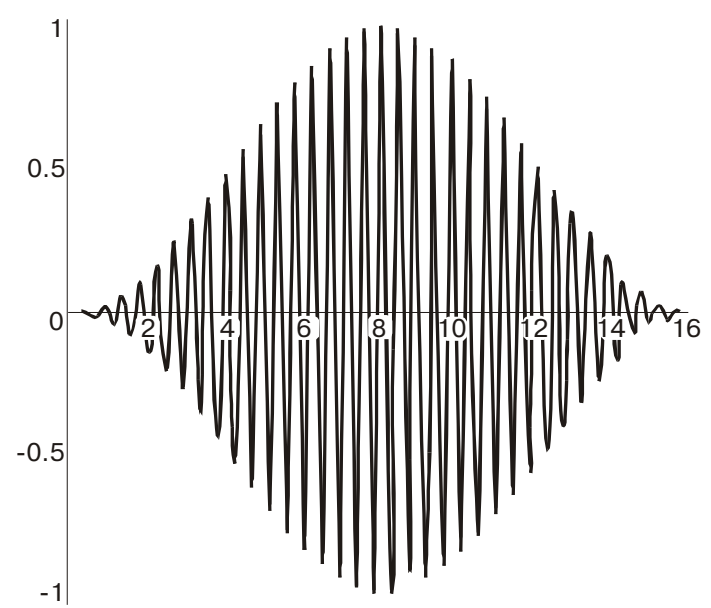

b)

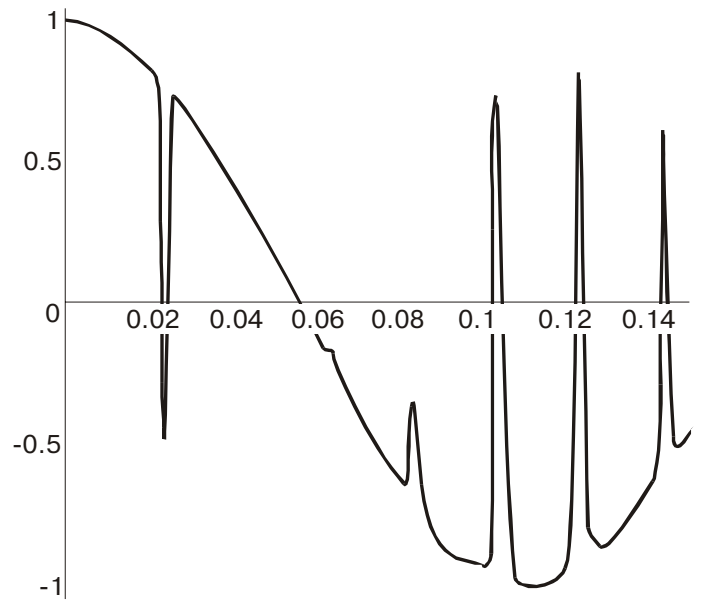

d)

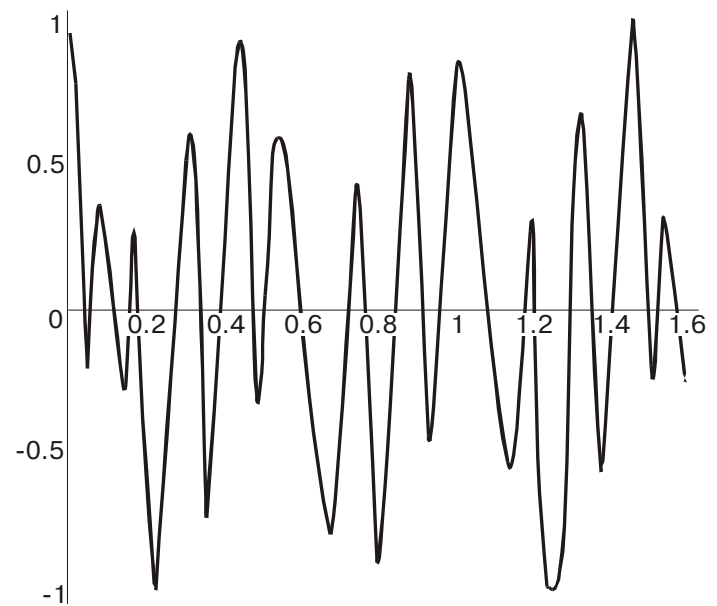

Fig. 4. The dependencies of the angular momentum $M_{z}$ (in relative units) and the polarization state $S_{3}$ (at the point vicinity $x=y=$ $0.2 \mu \mathrm{m})$ at the fiber length $z$ (in meters) of an inhomogeneous vortex in the wave state $|+1,-1\rangle$ for $(\mathrm{a}-\mathrm{b}) e=0.2, V=3.5$ and $(\mathrm{c}-\mathrm{d}) e=$ $0.01, V=3.5$ for a large ellipticity case.

tices (in according to J. Nye [34]). Thus we consider above all the general properties of the angular momentum of the vortex light fields.

\subsection{The field angular momenta in a weakly guiding fiber}

We specify the average density of the angular momentum of a light field in a media as:

$$
\langle\mathbf{M}\rangle=\frac{1}{2} \operatorname{Re}\left(\mathbf{r} \times \mathbf{D} \times \mathbf{B}^{*}\right),
$$

where $\mathbf{D}, \mathbf{B}$ are the vectors of electric and magnetic induction. Decomposing these vectors into parallel and perpendicular components (with respect to the optical axis), we obtain for the parallel component of the angular momentum density:

$$
\left\langle\mathbf{M}_{\|}\right\rangle=\frac{1}{2} \operatorname{Re}\left\{\boldsymbol{r}_{\perp} \times\left(\mathbf{D}_{\perp} \times \mathbf{B}^{*} \|+\mathbf{D}_{\|} \times \mathbf{B}^{*} \perp\right)\right\} .
$$

For electric component of guiding modes we have: $\mathbf{E}(x, y, z)=\mathbf{e}(x, y) e^{1 \beta z}$ and for magnetic component we have a similar representation. Following [30], express all the fields in (26a) in terms of the transverse electric field [15]

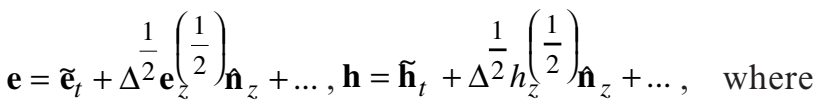

$$
\mathrm{e}_{z}^{\left(\frac{1}{2}\right)}=i \frac{\sqrt{2 \Delta}}{V} r_{0}\left(\nabla_{t} \cdot \mathrm{e}_{t}\right) \quad, \quad h_{z}^{\left(\frac{1}{2}\right)}=-i \frac{\sqrt{2 \Delta}}{V}\left(\frac{\varepsilon_{0}}{\mu_{0}}\right)^{\frac{1}{2}} \times
$$
$\times n_{c o} r_{0}\left(\nabla_{t} \times \widetilde{\mathbf{e}}_{t}\right)_{z}, \widetilde{\mathbf{h}}_{t}=n_{c o}\left(\frac{\varepsilon_{0}}{\mu_{0}}\right)^{\frac{1}{2}} \hat{\mathbf{n}}_{z} \times \widetilde{\mathbf{e}}_{t}$, the symbol $\left(\frac{1}{2}\right)$ specify the order of approximation in $\Delta$.Then in the first order in $\sqrt{\Delta}$ we have: 


\section{C.N. Alexeyev et al.: Spin-orbit interaction in a generic vortex field transmitted ...}

$$
\left\langle\mathbf{M}_{\|}^{\left(\frac{1}{2}\right)}\right\rangle=-m_{0} \operatorname{Im} \mathbf{r}_{\perp} \times\left(\widetilde{\mathbf{e}}_{\mathbf{t}} \times \nabla_{\mathbf{t}} \times \widetilde{\mathbf{e}}_{t}^{*}-\left(\nabla_{\mathbf{t}} \widetilde{\mathbf{e}}_{\mathbf{t}}\right) \widetilde{\mathbf{e}}_{\mathbf{t}}^{*}\right),
$$

where $m_{0}$ is defined in (18). For the total angular momentum in a cross-section after a little algebraic transformation we obtain [21]:

$$
\left\langle M_{z, t o t}^{\left(\frac{1}{2}\right)}\right\rangle=m_{0}\left\langle\Psi\left|\hat{l}_{z}+\sigma_{z}\right| \Psi\right\rangle
$$

If the field is a superposition of vortices:

$$
\begin{aligned}
& |\Psi\rangle=\sum_{i} c_{i}\left|\sigma_{i}, l_{i}\right\rangle \text {,then } \\
& \left\langle M_{z, t o t}^{\left(\frac{1}{2}\right)}\right\rangle=m_{0} \sum_{i}\left(l_{i}+\sigma_{i}\right)\left|c_{i}\right|^{2} .
\end{aligned}
$$

With the same assumptions we obtain for the total energy through a cross-section:

$$
\left\langle W_{t o t}^{(0)}\right\rangle=m_{0} \omega \sum_{j}\left|c_{j}\right|^{2}
$$

so that for pure states we easily obtain for the ratio «angular momentum/energy» a well known result $(l+\sigma) / \omega$ which describes both paraxial beams in a free space and in an inhomogeneous medium as well.

\subsection{The spin continuity and the spin momentum flux}

All optical processes observed usually in an experiment are perceived as a flux of certain physical value, intensity, in particular. An angular momentum is not exception. In our case, it takes place the continuous conversion of the orbital and spin angular momenta fluxes. Consider the equation governed by this process.

As it has been pointed out in [30] the matrix $\sigma_{z}$ (and the other two Pauli matrices) in the expression for the total angular momentum must not be regarded as a component of a spin angular momentum vector of the light. Nevertheless we would like to present a reason to justify the way of obtaining $S_{z}$ used in later. Consider Maxwell's equations for a homogeneous medium:

$$
\begin{aligned}
& \operatorname{rot} \mathbf{H}=\frac{\partial \mathbf{D}}{\partial t}, \quad \operatorname{rot} \mathbf{E}=-\frac{\partial \mathbf{B}}{\partial t}, \quad \operatorname{div} \mathbf{D}=0, \quad \operatorname{div} \mathbf{B}=0, \\
& \mathbf{D}=\varepsilon \mathbf{E}, \mathbf{B}=\mu \mathbf{H}
\end{aligned}
$$

and their complex conjugation. It is possible to obtain in vacuum the following equation:

$$
\begin{aligned}
& \frac{\partial}{\partial t}\left(\varepsilon_{0} \mathbf{E}^{*} \times \mathbf{E}+\mu_{0} \mathbf{H}^{*} \times \mathbf{H}\right)= \\
& =\left(\mathbf{E}^{*} \times \operatorname{rot} \mathbf{H}-\mathbf{H}^{*} \times \operatorname{rot} \mathbf{E}\right)-c . c .
\end{aligned}
$$

With the account of an evident relation: $(\mathbf{a} \times \operatorname{rot} \mathbf{b})_{i}=$ $=a_{j} \frac{\partial b_{j}}{\partial x_{i}}-\frac{\partial}{\partial x_{j}}\left(a_{j} b_{i}\right)$, where $\mathbf{a}, \mathbf{b}$ have the property: $d i v \mathbf{a}=d i v \mathbf{b}=0$, one can bring (26b) to the form of a continuity equation:

$$
\frac{\partial S_{i}}{\partial t}+\frac{\partial \Sigma_{i k}}{\partial x_{k}}=0
$$

where $\quad \mathbf{S}=-\frac{i}{2}\left(\varepsilon_{0} \mathbf{E}^{*} \times \mathbf{E}+\mu_{0} \mathbf{H}^{*} \times \mathbf{H}\right), \quad \Sigma_{i k}=-\frac{i}{2} \times$ $\times\left(\mathbf{E} \cdot \mathbf{H}^{*} \delta_{i k}+E_{k}^{*} H_{i}-H_{k}^{*} E_{i}+\right.$ c.c. . We interpret $\mathbf{S}$ as a vector of classical spin density, $\Sigma_{i k}-$ as a tensor of classical spin flux density. Note that $\mathbf{S}$ has a dimension of energy density and $\Sigma_{i k}-$ the one of Poynting's vector.

The expression for $\mathbf{S}$ is somewhat similar to the corresponding expressions for the spin angular momentum density in [30,32]. In vacuum the total spin momentum is proportional to the total spin angular momentum. For pure real fields (i.e. without phase shifts) $\mathbf{S}$ also equals zero, so it describes polarization properties of fields as well. But unlike the corresponding vectors in [30,32], $\mathbf{S}$ has local sense and is an integral of motion with a conservation law in the form of (26c). It should be noted that in the paraxial approximation the ratio of the total spin

flux $\left\langle\Pi_{3}\right\rangle=\iint_{S}\left\langle\Sigma_{33}\right\rangle d x d y$ to the total power is: $\frac{\left\langle\Pi_{3}\right\rangle}{\langle N\rangle}=\frac{\left\langle\Psi\left|\sigma_{z}\right| \Psi\right\rangle}{\langle\Psi \mid \Psi\rangle}$. The last relation provides an additional reason to identify $\sigma_{z}$ as an operator related to the spin properties of electromagnetic field.

\subsection{Conversion of the spin and orbital angular momenta in a fiber}

As evident the total spin angular momentum $S_{z}$ through a fiber cross-section can be found as $S_{z} \approx m_{0}\left\langle\Psi\left|\sigma_{z}\right| \Psi\right\rangle$ if we recognize $\sigma_{z}$ as the operator of the spin angular momentum. Then the total orbital angular momentum $L_{z}$ is found as $L_{z}=M_{z}-S_{z}$. With the account of these simple relations, we easily obtain for almost round fibers:

$S_{z} \approx m_{0} \cos \delta \beta_{24} z, \quad L_{z} \approx m_{0} \cos \delta \beta_{24} z$ 


\section{C.N. Alexeyev et al.: Spin-orbit interaction in a generic vortex field transmitted ...}

for $| \pm 1, \pm 1\rangle^{\text {ell }}$ vortices and

$S_{z} \approx m_{0} \cos \delta \beta_{13} z, \quad L_{z} \approx-m_{0} \cos \delta \beta_{13} z$

for $| \pm 1, \mp 1\rangle^{\text {ell }}$ vortices. As follows from (27).(28) at $\delta<\delta_{0}$ the transformation of the spin and orbital angular momentum takes place in a unified manner with the characteristic length $\Lambda_{1}$.

In highly elliptic fibers, angular momenta are transformed as follows:

$$
L_{z} \approx m_{0} \cos \left(\frac{D_{1}}{\widetilde{\beta}} z\right) \cos \left(\frac{B_{1}}{2 \widetilde{\beta}} z\right), S_{z} \approx m_{0} \cos \frac{B_{1}}{2 \widetilde{\beta}} z
$$

for $|1,1\rangle^{\text {ell }}$ vortex and

$$
L_{z} \approx-m_{0} \cos \left(\frac{D_{1}}{\widetilde{\beta}} z\right) \cos \left(\frac{B_{1}}{2 \widetilde{\beta}} z\right), S_{z} \approx m_{0} \cos \frac{B_{1}}{2 \widetilde{\beta}} z
$$

for $|1,-1\rangle^{\text {ell }}$ vortex. Fig. 5 gives the dependence curves. As follows from these expressions, two beating lengths $\Lambda_{1}$ and $\Lambda_{2}$ correspond to the scales on which the spin and orbital angular momentum change. Basically these two lengths are related to two existing mechanisms of the angular momentum transformation. The first one is connected with a birefringence of the form [29] and changes rather the orbital angular momentum than the spin one. This mechanism is responsible for the orbital angular momentum conversion in various mode converters $[7,26]$ and manifest itself on a relatively small length $\Lambda_{2}$. The other mechanism is connected with the so-called topological birefringence [25] and exhibit itself on larger scales characterized by $\Lambda_{1}$. This mechanism is also immanently connected with the spin-orbit interaction [23,24]. The obtained behavior of the angular momenta is consistent with our expectations. As it was demonstrated in [30], the orbital angular momentum changes in locally isotropic media with violated axial symmetry, while the spin angular momentum is changed by media's optical activity. The refined perturbation theory [31] reveals large-scale variation of radiation's ellipticity (and thus its spin angular momentum) even in locally isotropic media. The obtained curves demonstrate just these predicted features.

Interesting results are obtained when the transformation of initially polarized light in elliptic fibers is considered. If we launch into the fiber a linearly polarized radiation, so that the boundary condition is written as $\left.|L P\rangle^{e l l}\right|_{z=0}=\frac{1}{\sqrt{2}}(|1, l\rangle+|-1, l\rangle)$, its distribution in the case $\delta<<\delta_{0}$ is given by: a)

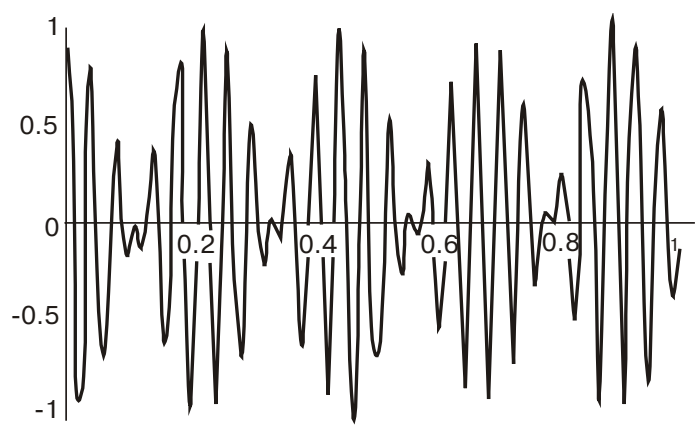

c)

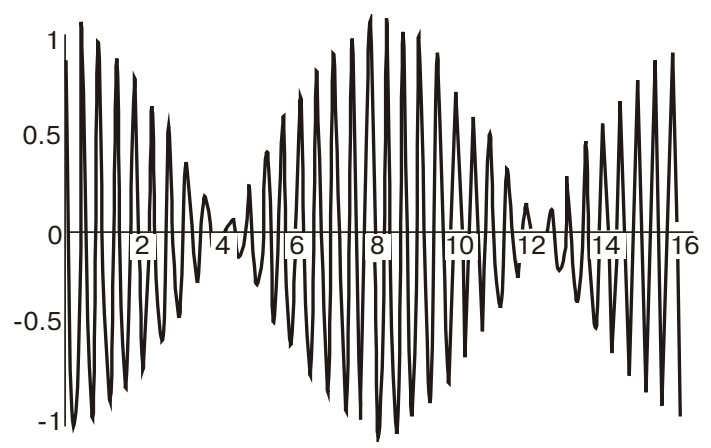

b)

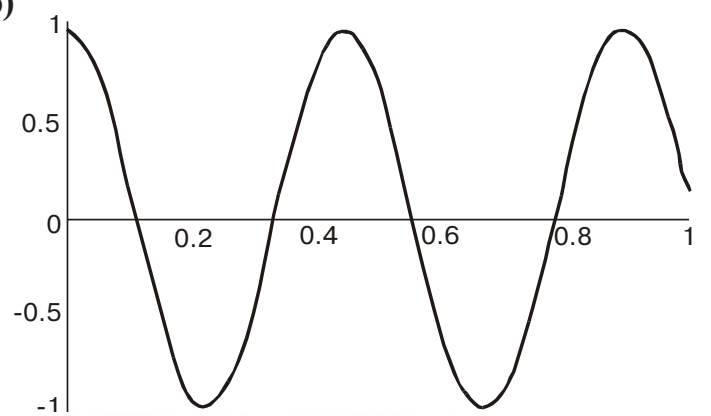

d)

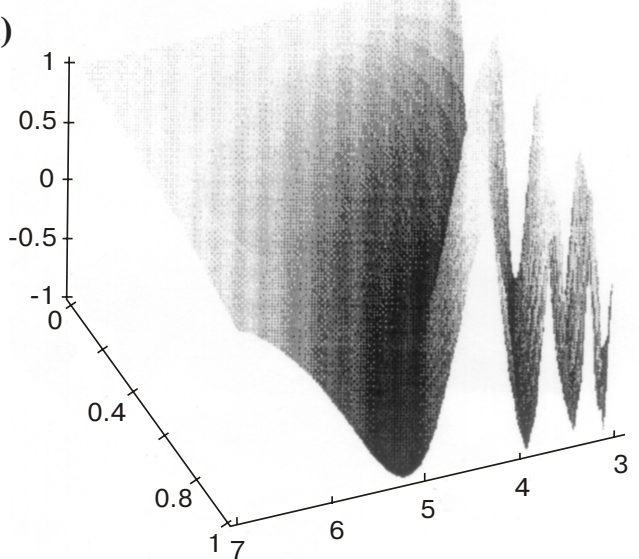

Fig. 5. The dependencies of the angular orbital $L_{z}(\mathrm{a}, \mathrm{c})$ and spin $S_{z}(\mathrm{~b}, \mathrm{~d})$ momenta of inhomogeneous vortex in the state $|+1,-1\rangle$ at a fiber length $z$ (in meters) and a waveguiding parameter $V$ (in (a,b,c) $V=3.5$ ) for $e=0.2(\mathrm{a}, \mathrm{b}, \mathrm{d})$ and $e=0.01(\mathrm{c})$. 


\section{C.N. Alexeyev et al.: Spin-orbit interaction in a generic vortex field transmitted ...}

$$
\begin{aligned}
& |L P\rangle^{e l l}=\frac{1}{2 \sqrt{2}}\left[|1, l\rangle\left(e^{i \beta_{2} z}+e^{i \beta_{4} z}\right)+\right. \\
& +|-1,-l\rangle\left(e^{i \beta_{4} z}-e^{i \beta_{2} z}\right)+|1,-l\rangle\left(e^{i \beta_{3} z}-e^{i \beta_{1} z}\right)+ \\
& \left.+|-1, l\rangle\left(e^{i \beta_{3} z}+e^{i \beta_{1} z}\right)\right],
\end{aligned}
$$

while in the case $\delta>\delta_{0}$ the field distribution is:

$$
\begin{aligned}
& |L P\rangle^{e l l}=\frac{1}{2 \sqrt{2}}\left[|1, l\rangle\left(e^{i \beta_{2} z}+e^{i \beta_{3} z}\right)+\right. \\
& +|-1,-l\rangle\left(e^{i \beta_{3} z}-e^{i \beta_{2} z}\right)+|1,-l\rangle\left(e^{i \beta_{3} z}-e^{i \beta_{2} z}\right)+ \\
& \left.+|-1, l\rangle\left(e^{i \beta_{2} z}+e^{i \beta_{3} z}\right)\right] .
\end{aligned}
$$

Then for the angular momenta we obtain:

$$
\begin{aligned}
& M_{z} \approx S_{z} \approx m_{0} \cos \delta \beta_{24} z, \quad L_{z} \approx 0 \quad\left(\delta<<\delta_{0}\right), \\
& M_{z} \approx S_{z} \approx m_{0} \cos \delta \beta_{13} z, \quad L_{z} \approx 0 \quad\left(\delta<<\delta_{0}\right)
\end{aligned}
$$

for $l=1$. If we excite the fiber with a circularly polarized radiation, the boundary condition assumes the form:

$$
\left.|C P\rangle^{\text {ell }}\right|_{z=0}=\frac{1}{\sqrt{2}}(|1, l\rangle+|1,-l\rangle) \text {. Then the field distribu- }
$$

tion is given by:

$$
\begin{aligned}
& |C P\rangle^{e l l}=\frac{1}{2 \sqrt{2}}\left[|1, l\rangle\left(e^{i \beta_{2} z}+e^{i \beta_{4} z}\right)+\right. \\
& +|-1,-l\rangle\left(e^{i \beta_{4} z}-e^{i \beta_{2} z}\right)+|1,-l\rangle\left(e^{i \beta_{1} z}+e^{i \beta_{3} z}\right)+ \\
& \left.+|-1, l\rangle\left(e^{i \beta_{3} z}-e^{i \beta_{1} z}\right)\right]
\end{aligned}
$$

in the case of small ellipticity and:

$$
\begin{aligned}
& |C P\rangle^{e l l}=\frac{1}{2 \sqrt{2}}\left[|1, l\rangle\left(e^{i \beta_{1} z}+e^{i \beta_{3} z}\right)+\right. \\
& +|-1,-l\rangle\left(e^{i \beta_{3} z}-e^{i \beta_{1} z}\right)+|1,-l\rangle\left(e^{i \beta_{1} z}+e^{i \beta_{3} z}\right)+ \\
& \left.+|-1, l\rangle\left(e^{i \beta_{3} z}-e^{i \beta_{1} z}\right)\right]
\end{aligned}
$$

in the case of large ellipticity. For the angular momenta we obtain:

$$
\begin{aligned}
& M_{z} \approx L_{z} \approx m_{0} \cos \delta \beta_{24} z, \quad S_{z} \approx 0 \quad\left(\delta<<\delta_{0}\right), \\
& M_{z} \approx L_{z} \approx m_{0} \cos \delta \beta_{23} z, \quad S_{z} \approx 0 \quad\left(\delta<<\delta_{0}\right) .
\end{aligned}
$$

From the expressions for polarization corrections given in the Tables 1,2 we conclude that in the both cases the spin angular momentum oscillates on large lengths. The orbital angular momentum possess small beating length in the case $\delta \gg \delta_{0}$, while at $\delta \gg \delta_{0}$ it also varies at large distances.

\section{Conclusions}

We have studied the propagation of the simplest possible vortical fields in elliptic fibers. To make it we obtained the eigenfunctions of the operator of a spin-orbit interaction and the spectrum of polarization corrections to the scalar propagation constant in the case of relatively large and small values of a fiber ellipticity. The obtained result revealed certain discordance with the spectra of polarization corrections given in [15], namely in the case of a small fiber ellipticity. We explain this discordance as follows. The results of [15] are valid only if we treat the spin-orbit interaction as a small perturbation on the background of large deformation-induced effective interaction, included in the structure of the $\hat{H}_{0}$ unperturbed Hamiltonian. If these two interactions are comparable or the latter is much less, we must use the expressions obtained in the present paper. The basic difference between the perturbation theory developed in [15] and the one suggested in the present paper is in the symmetry of the ground state. While in [15] the ground state is twotimes degenerated, the degeneration degree in our case equals four. This is also the basic reason of the drastic difference in vortex evolution in weakly elliptic and strongly elliptic fibers.

As we have established, both homogeneous and inhomogeneous vortices propagating in an elliptic fiber undergo a mode conversion. Unlike lens converters [33], en elliptic fiber converts both topological charge and polarization of vortices observing in a cross-section. But while in strongly elliptic fibers the mode conversion patterns are somewhat similar to that observed in lens converters, a weakly elliptic fiber exhibits the unique type of conversion not existing in lens converters. This type of conversion is characterized with larger conversion length $\Lambda_{1} \propto e^{4}$, so those fibers with the length $L \ll \Lambda_{1}$ will transmit the vortex in an almost unchanged form. This prediction could not have been made on the basis of relations obtained in [15] and constitutes one of the main results of the present paper. The difference in the behavior of vortices in strongly and weakly elliptic fibers is so striking that one can even speak on two different physical phases, which are realized in deformed fibers and characterized by different types of symmetry. It should be noticed that in an ideal circular fiber, the vortex ground state is not degenerated with respect to a polarization [24] only.

\section{References}

1. J.F. Nye, M.V. Berry Dislocation in wave trains // Proc. R. Soc. Lond. A 333, pp. 165-190 (1974).

2. I.V. Basisty, V.Yu. Bazhenov, M.S. Soskin, M.V. Vasnetsov Optics of light beams with screw dislocations // Optics Comm. 103, pp. 422-528 (1993).

3. M.S. Soskin, V.N. Gorshkov, M.V. Vasnetsov, J.T. Malos, N.R. Heckenberg //Topological charge and angular momentum of light carrying optical vortices // Phys. Rev. A 56(5), pp. 4064-4075 (1998).

4. E. Abramochkin, V. Volostnicov Spiral type beams: optical and quantum aspects // Optics Comm. 125, pp.302-323 (1996). 


\section{C.N. Alexeyev et al.: Spin-orbit interaction in a generic vortex field transmitted ...}

5. M. Harris, C.A. Hill, J.M. Vaughman Optical helices and spiral interference fringes // Optics Comm. 106, pp. 161-166 (1994).

6. Z.S. Sacks, D. Rozas, G.A. Swartzlander Holografic formation of optical-vortex filament // Journ. Opt. Soc. Am. B 15(8), pp. 2226-2234 (1998).

7. L. Allen, M.W. Beijersbergen, R.J.C. Spreeuw, J.P. Woerdman Orbital angular momentum of light and the transformatiom of Laguerre-Gaussian laser modes // Physical Review A 45(11), pp. 8185-8189 (1992).

8. R.A. Beth Mechanical detection and measurement of the angular momentum of light // Physical Review 50, pp. 115-125 (1936).

9. H. He, M.E.J. Friese, N.R. Heckenberg, H. RubinszteinDunlop Direct observation of transfer of angular momentum to absorptive particles from a laser beam with a phase singularity // Phys. Rev. Letters 75(5), pp.826-829 (1995).

10. A.V. Volyar, V.G. Shvedov, T.A. Fadeyeva // Techn. Phys. Journ. Lett. 25(5), (1999)

11. J.F. Nye, Polarization effects in the diffraction of electromagnetic waves: the role of disclinations // Proc. R. Soc. Lond. A 387, pp. 105-132. (1983).

12. J.F. Nye, Lines of circular polarization in electromagnetic wave fields // Proc. R. Soc. Lond. A 389, pp.279-290 (1983)

13. J.F. Nye, J.V. Hajnal, The wave structure of monochromatic electromagnetic radiation // Proc. R. Soc. Lond. A 409, pp. 21-36 (1987)

14. N.B. Baranova, B.Ya. Zel'dovich Wavefront dislocations and amplitude zeros // Exp. and Theor. Phys. Journ. 80, pp. 1789 1794 (1981) (Rush).

15. A.W. Snyder, J.D. Love, Optical Waveguide Theory. Chapman and Hall, London, New York, (1985)

16. M.Ya. Darsht, B.Ya. Zel'dovich, I.V.Kotievsky, N.L. Kundikova. Wave front single dislocation formation// Journ. Theor. and Exp. Phys. 107(5), pp.1467-1472, (1995. ) (Rush).

17. A.V. Volyar, T.A. Fadeyeva Mode vortex nature in an optical fiber: II. An optical vortex propagation// Techn. Phys. Journ. Lett. 22(8), P. 63-67 (1996) (Rush).

18. A.V. Volyar, T.A. Fadeyeva Optics of singularities of a lowmode fiber: I. Circular disclinations // Optics and Spectroscopy 85(2), pp. 264-271 (1998).

19. A.V. Volyar, T.A. Fadeyeva Optics of singularities of a lowmode fiber: II. Optical vortices // Optics and Spectroscopy 85(2), pp. 272-280 (1998).
20. K.N. Alexeyev, T.A. Fadeyeva, A.V. Volyar, M.S. Soskin, Optical vortices and the flow of their angular momentum in a multimode fiber // Semiconductor Physics. Quantum Electronics \& Optoelectronics 1(1), pp. 1-8 (1998).

21. C.N. Alexeyev, Yu.A. Fridman, A.N. Alexeyev, Angular momentum and spin-orbit interaction in weakly guiding fibers // Ukr. Fiz. Zh., 44(6), pp. 74-81 (1999).

22. A.V. Volyar, T.A. Fadeyeva Optical vortices in low-mode fiber: 1. Angular momentum of field // Optics and Spectroscopy 86(2), pp.242-250. (1999).

23. V.S. Liberman, B.Ya. Zel'dovich, Spin-orbit interaction of a photon in an inhomogeneous medium // Phys. Rev. A 45(8), pp. 5199-5207 (1992).

24. A.V. Volyar, V.Z. Zhilaitis, V.G. Shvedov Optical eddies in small-mode fibers: 2. The spin-orbit interaction // Optics and Spectroscopy, 86(4), pp. 664-670. (1999).

25. A.V. Volyar, V.Z. Zhilaitis, V.G. Shvedov, M.S. Soskin, T.A Fadeyeva, Topological birefringence of optical vortices in an inhomogeneous media // Optics of Atmosphere and Ocean 11(11), pp. 1199-1214 (1998) (Rush).

26. E. Abramochkin, V. Volostnikov Beam transformations and nontransformed beams // Optics Comm. 83(1,2), pp. 123-135.

27. Jun-ichi Sakai, Tatsuya Kimura, Birefringence and polarization characteristics of single-mode optical fiber under elastic deformations // IEEE Journ. Quant. Electron 17(6), pp. 1041$1051(1981)$

28. A.W. Snyder, W.R. Young, Modes of optical fiber // Journ. Opt. Soc. Am. 68(3), pp. 298-309. (1978).

29. M. Born, E. Wolf, Principles of Optics, $6^{\text {th }}$ ed., Pergamon, Oxford, (1987).

30. M.V. Berry, Paraxial beams of spinning light // Proceedings of SPIE 3487, p. 6 (1997).

31. A.Yu. Savchencko, B.Ya. Zel'dovich, Wave propagation in a guiding structure: one step beyond the paraxial approximation // Journ. Opt. Soc. Am. 13(2), pp. 273-281, (1996).

32. L.D.Landau, E.M.Lifshitz, L.P. Pitaevsky, Quantum Mechanics, $2^{\text {nd }}$ ed., Pergamon, Oxford (1984).

33. M.W. Beijersbergen, L. Allen, van der Veen H.E.L.O., J.P. Woerdman, Astigmatic laser mode converters and transfer of orbital angular momentum // Opt. Comm. 196, pp. 123-132. (1993).

34. J.F. Nye, Natural Focusing and Fine Structure of Light, Institute of Physics Publishing Bristol and Philadelphia, (1999). 